\title{
The interaction of HSV-1 tegument proteins pUL36 and pUL37: a novel target for antivirals that inhibit viral assembly
}
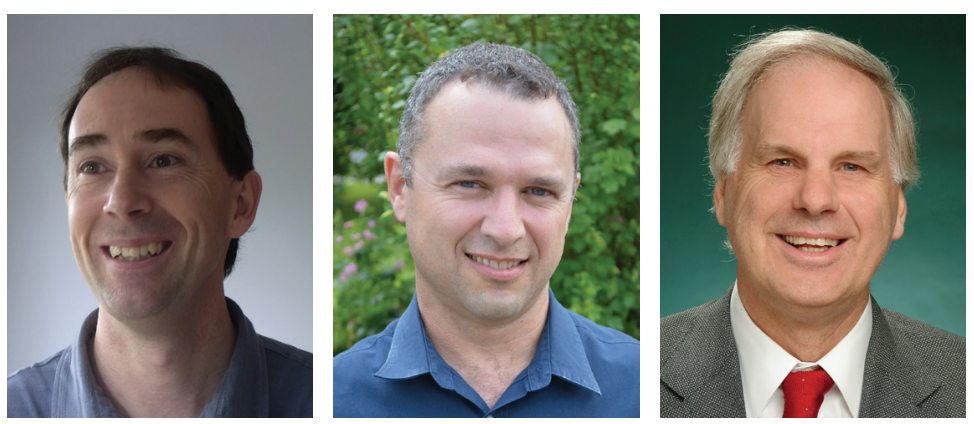

\author{
“Given that interacting homologues of \\ pUL36 and pUL37 are present across the \\ Herpesviridae family ... the opportunity exits \\ for developing a generic antiviral drug that \\ targets their interaction.”
}

Russell J Diefenbach ${ }^{* 1}$, Cornel Fraefel ${ }^{2} \&$ Anthony L Cunningham ${ }^{1}$

Infection with human pathogens herpes simplex virus type 1 (HSV-1) or the closely related HSV-2 is for life. The herpes simplex viruses infect orogenital stratified squamous epithelium, then enter cutaneous nerves and are transported to the dorsal root ganglion where they remain latent for life. Periodic reactivation and transport back along the same nerves leads to recurrent disease close to the site of initial infection or asymptomatic shedding of virus in secretions. Infection causes ocular disease or encephalitis in immunocompromised adults and severe disseminated infection and encephalitis with high mortality and neurological impairment in neonates. Over one third of the world's population suffer from recurrent HSV infections several times a year and are thus capable of transmitting HSV by close personal contact.

Some of the most effective and nontoxic antiviral drugs currently available, acyclovir (Zovirax), valaciclovir (Valtrex) and famiciclovir, are used for treating herpes simplex and varicella zoster virus infections. They have been modelled upon the discovery of nucleosides with antiviral activity isolated from sea sponges such as Cryptotethya crypta. These selective DNA polymerase inhibitors belong to the class of nucleoside analogues, but instead of ribose they contain an open polyol structure. The mechanism of action involves progressive phosphorylation by viral then cellular thymidine kinases. The end active metabolite, acycloguanosine triphosphate, has approximately 100-times higher affinity towards viral polymerase than the cellular enzyme. Thus, it is preferentially incorporated into the viral DNA and because it lacks a 3' end no more nucleotides can be added, resulting in premature chain termination [1].

Extensive clinical use of these drugs has led to the emergence of resistant viral strains.

\section{KEYWORDS}

- antiviral $\bullet$ herpes simplex virus - pUL36 • pUL37 • tegument • virus assembly

\footnotetext{
'Centre for Virus Research, Westmead Millennium Institute, The University of Sydney \& Westmead Hospital, Westmead, NSW 2145, Australia

${ }^{2}$ Institute of Virology, University of Zurich, 8057 Zurich, Switzerland

*Author for correspondence: russell.diefenbach@sydney.edu.au
}

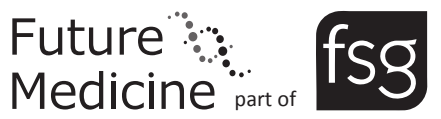


“...the targeting of different stages of

herpesviral replication would allow combination with current drugs ... and aid in more rapid resolution of disease and minimise antiviral resistance..."
While resistance is not a serious issue for immunocompetent individuals, it is a serious issue for immunocompromised patients, especially those with HIV or for those who have undergone organ transplantation. Furthermore, these drugs reduce the frequency and magnitude of viral shedding of HSV-2 from the anogenital tract of infected women but frequent breakthrough episodes occur, probably due to their short half life [2]. This is important, as there is strong evidence that prior infection with HSV-2 increases HIV acquisition, and reactivation of HSV-2 increases HIV replication and transmission [3]. Attempted suppression of genital HSV-2 shedding with these drugs has not prevented HIV acquisition [4]. Given that no effective vaccine is currently available for HSV, there is a crucial need for the development of new anti-herpes drugs with a longer half-life that can inhibit mucosal infection and shedding by both wild-type viruses and drug-resistant strains, especially to prevent asymptomatic transmission and to reduce HIV acquisition and transmission [3]. Clearly, novel nontoxic drugs that are more efficacious and target different viral functions than those presently available would be of great use, especially for resistant viruses in immunocompromised patients. In particular, the targeting of different stages of herpesviral replication would allow combination with current drugs (which all have the same site of action) and aid in more rapid resolution of disease and minimise antiviral resistance [1].

In molecular herpesvirus research there has been a shift away from the well characterized viral capsid proteins and glycoproteins towards the less well-characterized viral tegument (bridges capsid and glycoprotein envelope) and its role in viral replication [5]. A number of studies, including our own, have now identified the interaction of tegument proteins pUL36 and pUL37 and their homologues across the Herpesviridae family [6-10]. The interaction of pUL36 with capsid proteins provides the platform for subsequent recruitment of remaining tegument, including pUL37 [11].

For HSV-1 and the related swine pathogen Pseudorabies virus $(\mathrm{PrV})$, deletion of the genes encoding either of pUL36 or pUL37 blocks secondary envelopment in cell lines (process reviewed in [12]) of capsid and subsequent virion maturation [11,13-17]. In the case of HSV-1, residue D631 in the C-terminal pUL36 binding domain of pUL37 is essential for binding pUL36 [18]. Deletion of the 25 amino acid pUL37-binding domain, located in the N-terminus of HSV-1 pUL36 [19], revealed that the interaction of pUL36 and pUL37 is essential for the process of secondary envelopment during virion assembly [20]. The interaction of pUL36 and pUL37, which forms the inner tegument layer adjacent to the viral capsid, appears to be important for both trafficking to the site of secondary envelopment $[11,17,20]$, as well as subsequent recruitment of remaining viral components through direct directions with either tegument or envelope proteins [21,22].

Recently, the structure of the N-terminal half of $\operatorname{PrV}$ pUL37 has been elucidated and appears to resemble components of multi-subunit tethering complexes that regulate vesicle trafficking in cells [23]. This is consistent with a role in trafficking during viral assembly, but whether this occurs in conjunction with binding of pUL36, via the C-terminal half of pUL37, and/or other host cell proteins remains to be determined. The structure of full-length pUL37 or the C-terminal half of pUL37, which contains the pUL36 binding domain [18,24], has not yet been reported. It may well be that the presence of bound pUL36 results in conformational changes in pUL37, which enhances stability of the two proteins. Preliminary data from our laboratory does suggest that coexpression in bacteria of a minimal fragment of pUL36, containing the pUL37 binding domain, and the C-terminal half of pUL37 results in enhanced expression levels of pUL37. Certainly, there is increasing evidence that pUL36 is also stabilized by bound pUL37, which may prevent proteasomal degradation of pUL36 during the course of infection $[18,20]$.

The growing body of knowledge on the nature and essential role of the interaction of pUL36 and pUL37 in HSV assembly now provides a strong basis for developing therapeutics that block the assembly of HSV. Successful soluble expression of pUL36 and pUL37 interacting domains, and subsequent knowledge of the structure of a complex of pUL36 and pUL37 would be a significant step in aiding the discovery of small molecule inhibitors that block viral assembly. This would represent a different strategy to the current inhibitors of HSV DNA replication, which include those that either directly or indirectly target viral DNA polymerase and those in clinical trials that inhibit viral helicase-primase [1]. It would also allow combination therapy with currently available antiviral drugs (all of which have the same mode of action) aimed at different stages of the HSV replication cycle, similar to 
that for HIV and hepatitis B virus. In addition to targeting assembly, the development of inhibitors of HSV entry also provides much needed diversity in drug design [25].

Given that interacting homologues of pUL36 and pUL37 are present across the Herpesviridae family (reviewed in [5]), the opportunity exits for developing a generic antiviral drug that targets their interaction. Although the overall amino acid homology between homologues is relatively small, the probability of structural similarity for interacting domains is likely to be high, given they have similar roles in viral assembly. Due to the conservation of the interaction of pUL36 with pUL37, knowledge gained with HSV and PrV will also facilitate our understanding of assembly across the Herpesviridae family. Furthermore, it will provide essential information for development of attenuated $\mathrm{HSV}$ vaccine candidates.

\section{Financial \& competing interests disclosure}

This work was supported by grant 632616 from the Australian National Health and Medical Research Council. The authors have no other relevant affiliations or financial involvement with any organization or entity with a financial interest in or financial conflict with the subject matter or materials discussed in the manuscript apart from those disclosed.

No writing assistance was utilized in the production of this manuscript.

\section{References}

1 Vere Hodge RA, Field HJ. Antiviral agents for herpes simplex virus. Adv. Pharmacol. 67, 1-38 (2013).

2 Schiffer JT, Swan DA, Corey L, Wald A. Rapid viral expansion and short drug half-life explain the incomplete effectiveness of current herpes simplex virus 2-directed antiviral agents. Antimicrob. Agents Chemother. 57, 5820-5829 (2013).

3 Van de Perre P, Segondy M, Foulongne V et al. Herpes simplex virus and HIV-1: deciphering viral synergy. Lancet Infect. Dis. 8, 490-497 (2008).

4 Celum C, Wald A, Lingappa JR et al. Acyclovir and transmission of HIV-1 from persons infected with HIV-1 and HSV-2. N. Engl. J. Med. 362, 427-439 (2010).

5 Kelly BJ, Fraefel C, Cunningham AL, Diefenbach RJ. Functional roles of the tegument proteins of herpes simplex virus type 1. Virus Res. 145, 173-186 (2009).

6 Vittone V, Diefenbach E, Triffett D, Douglas MW, Cunningham AL, Diefenbach RJ. Determination of interactions between tegument proteins of herpes simplex virus type 1.J. Virol. 79, 9566-9571 (2005).

7 Rozen R, Sathish N, Li Y, Yuan Y. Virionwide protein interactions of Kaposi's sarcoma-associated herpesvirus. J. Virol. 82, 4742-4750 (2008).

8 Stellberger T, Häuser R, Baiker A, Pothineni VR, Haas J, Uetz P. Improving the yeast two-hybrid system with permutated fusions proteins: the Varicella Zoster virus interactome. Proteome Sci. 8, 8 (2010).

9 To A, Bai Y, Shen A et al. Yeast two hybrid analyses reveal novel binary interactions between human cytomegalovirus-encoded virion proteins. PLoS ONE 6, e17796 (2011).

10 Klupp BG, Fuchs W, Granzow H, Nixdorf R, Mettenleiter TC. Pseudorabies virus UL36 tegument protein physically interacts with the UL37 protein. J. Virol. 76, 3065-3071 (2002).

11 Sandbaumhüter M, Döhner K, Schipke J et al. Cytosolic herpes simplex virus capsids not only require binding inner tegument protein pUL36 but also pUL37 for active transport prior to secondary envelopment. Cell. Microbiol. 15, 248-269 (2013).

12 Mettenleiter TC, Klupp BG, Granzow H. Herpesvirus assembly: an update. Virus Res. 143, 222-234 (2009)

13 Desai P, Sexton GL, McCaffery JM, Person S. A null mutation in the gene encoding the herpes simplex virus type 1 UL37 polypeptide abrogates virus maturation. J. Virol. 75, 10259-10271 (2001).

14 Desai PJ. A null mutation in the UL36 gene of herpes simplex virus type 1 results in accumulation of unenveloped DNA-filled capsids in the cytoplasm of infected cells. J. Virol. 74, 11608-11618 (2000).

15 Klupp BG, Granzow H, Mundt E, Mettenleiter TC. Pseudorabies virus UL37 gene product is involved in secondary envelopment. J. Virol. 75, 8927-8936 (2001).

16 Fuchs W, Klupp BG, Granzow H, Mettenleiter TC. Essential function of the pseudorabies virus UL36 gene product is independent of its interaction with the UL37 protein. J. Virol. 78, 11879-11889 (2004).

17 Pasdeloup D, Beilstein F, Roberts APE, McElwee M, McNab D, Rixon FJ. Inner tegument protein pUL37 of herpes simplex virus type 1 is involved in directing capsids to the trans-Golgi network for envelopment. J. Gen. Virol. 91, 2145-2151 (2010).
18 Kelly BJ, Mijatov B, Fraefel C, Cunningham AL, Diefenbach RJ. Identification of a single amino acid residue which is critical for the interaction between HSV-1 inner tegument proteins pUL36 and pUL37. Virology 422, 308-316 (2012).

19 Mijatov B, Cunningham AL, Diefenbach RJ. Residues F593 and E596 of HSV-1 tegument protein pUL36 (VP1/2) mediate binding of tegument protein pUL37. Virology 368, 26-31 (2007).

20 Kelly BJ, Bauerfeind R, Binz A et al. The interaction of the HSV-1 tegument proteins pUL36 and pUL37 is essential for secondary envelopment during viral egress. Virology 454-455, 67-77 (2014).

21 Jambunathan N, Chouljenko D, Desai P et al. Herpes simplex virus 1 protein UL37 interacts with viral glycoprotein $\mathrm{gK}$ and membrane protein UL20 and functions in cytoplasmic virion envelopment. J. Virol. 88, 5927-5935 (2014).

22 Svobodova S, Bell S, Crump CM. Analysis of the interaction between the essential herpes simplex virus 1 tegument proteins VP16 and VP1/2.J. Virol. 86, 473-483 (2012).

23 Pitts JD, Klabis J, Richards AL, Smith GA, Heldwein EE. Crystal structure of the herpesvirus inner tegument protein UL37 supports its essential role in control of viral trafficking. J. Virol. 88, 5462-5473 (2014).

24 Bucks MA, Murphy MA, O’Regan KJ, Courtney RJ. Identification of interaction domains within the UL37 tegument protein of herpes simplex virus type 1 . Virology 416 , 42-53 (2011).

25 Hadigal S, Shukla D. Exploiting herpes simplex virus entry for novel therapeutics. Viruses 5, 1447-1465 (2013). 